\title{
Systematic review and meta-analysis of the therapeutic effects of minimally invasive transforaminal interbody fusion on spondylolisthesis
}

\author{
Zaifei Chen ${ }^{1}$, Wenxu Wu ${ }^{1}$, Huazhang Xiong ${ }^{2}$, Guodong Li $^{1}$, Wenbo Zhang ${ }^{1}$, Yingyin Gao ${ }^{1}$, \\ Mingchang Wang ${ }^{1}$
}

${ }^{1}$ Department of Orthopedics, Zunyi Hospital of Traditional Chinese Medicine, Zunyi, China; ${ }^{2}$ Department of Orthopedics, The First Department of Orthopedics, Affiliated Hospital of Zunyi Medical University, Zunyi, China

Contributions: (I) Conception and design: Z Chen, M Wang; (II) Administrative support: W Wu, G Li; (III) Provision of study materials or patients: Z Chen, W Wu, H Xiong, G Li, M Wang; (IV) Collection and assembly of data: All authors; (V) Data analysis and interpretation: Z Chen, H Xiong, W Zhang, Y Gao; (VI) Manuscript writing: All authors; (VII) Final approval of manuscript: All authors.

Correspondence to: Mingchang Wang. Department of Orthopedics, Zunyi Hospital of Traditional Chinese Medicine, Crossing of Donglian Line 2 and Hexing Avenue, Honghuagang District, Zunyi, China. Email: 494896335@qq.com.

\begin{abstract}
Background: Minimally invasive transforaminal interbody fusion (MI-TLIF) can minimize surgical incision, tissue damage, and intraoperative blood loss in the treatment of spondylolisthesis. However, there is a lack of evidence-based research to confirm its clinical efficacy.

Methods: Chinese and English databases were searched with "open", "minimally invasive transforaminal interbody fusion", "MIS-TLIF", "spondylolisthesis", and "open transforaminal interbody fusion" as search terms. Rev Man 5.3 provided by the Cochrane system was used to assess the quality of the literature.

Results: Of the 12 randomized controlled trials (RCTs), 7 references were level A (58.34\%), 4 were B level (33.33\%), and 1 reference was C level (8.33\%). There was a statistically significant difference in intraoperative blood loss between MI-TLIF and open transforaminal interbody fusion (O-TLIF) in the treatment of spondylolisthesis [mean difference $(M D)=-349.35,95 \%$ confidence interval (CI): $(-410.66$, -288.03), $\mathrm{P}<0.00001]$. There was also a statistically significant difference in visual analogue scale (VAS) scores before and after MI-TLIF at the last follow-up [MD =5.72, 95\% CI: $(4.83,6.62), \mathrm{P}<0.00001]$, and in the complication rate between MI-TLIF and O-TLIF [odds ratio (OR) $=0.48,95 \%$ CI: $(0.30,0.76)$, $\mathrm{P}<0.00001]$.

Discussion: This meta-analysis confirmed that MI-TLIF could significantly reduce intraoperative blood loss, mitigate patient pain, and reduce the incidence of complications without increasing the operation time in the treatment of spondylolisthesis.
\end{abstract}

Keywords: Minimally invasive transforaminal interbody fusion (MI-TLIF); orthopedics; spondylolisthesis

Submitted Jul 20, 2021. Accepted for publication Sep 02, 2021.

doi: 10.21037/apm-21-2137

View this article at: https://dx.doi.org/10.21037/apm-21-2137

\section{Introduction}

Spondylolisthesis is a degenerative disease of the spine arising from abnormal connection between adjacent vertebral bodies so that the upper vertebral body is partially or completely slipped relative to the lower vertebral body. It is common in middle-aged and elderly people (1).
At present, there are mainly two types of transforaminal interbody fusion (TLIF). One is open transforaminal interbody fusion (O-TLIF), and the other is minimally invasive transforaminal interbody fusion (MI-TLIF) (2). Of the two, O-TLIF has been used extensively and is favored by scholars because it involves less surgical trauma, less 
blood loss, and faster recovery time. However, it is likely to cause problems such as a loss of intervertebral motion and accelerated adjacent vertebrae degeneration (3). MITLIF was first proposed by Foley in 2002 (4), and during this minimally invasive procedure, a damaged disc in the lumbar spine is replaced with an expandable implant, and hardware to stabilize the spine. This procedure relieves nerve root compression, which is a common cause of pain in back and legs (5). The surgeon inserts a needle into the back, and carefully passes it through a natural opening in the spine called a foramen, reducing tissue damage and preserving bone. The needle is pushed into the target disc, a guide wire is passed through the needle, and the needle is removed. The guide wire is used to direct a dilator, and then a metal tube into the disc, creating a larger opening for implanting (6). At present, the technique of spinal fusion is recognized as the "gold standard" for the treatment of spondylolisthesis (7).

The short-term efficacy of MI-TLIF in the treatment of spondylolisthesis was analyzed. It did not require dissection of the paraspinal muscle and caused little muscle damage. The postoperative pain experience of patients was relatively mild and the functional exercise time was advanced, which was conducive to rapid recovery. However, the longterm efficacy of MI-TLIF is still controversial. Lau et al. [2013] (8) showed that there was no significant difference between the long-term effect of mi-TLIF and posterior interbody fusion. Patients can benefit from MI-TLIF in many ways. A minimally invasive approach reduces time in the operating room and minimizes blood loss and tissue damage. It helps minimize pain during healing and also speeds recovery time and reduces the chance for complications. However, the efficacy of MI-TLIF and O-TLIF has not been analyzed systematically.

Existing research is mostly single-center randomized controlled trials (RCTs) with small sample sizes. In this study, a meta-analysis was conducted to compare the efficacy of MITLIF and O-TLIF, to provide a scientific evidence-based platform for the clinical treatment of spondylolisthesis.

We present the following article in accordance with the PRISMA reporting checklist (available at https://dx.doi. org/10.21037/apm-21-2137).

\section{Methods}

\section{Literature retrieve}

With "open", "minimally invasive transforaminal interbody fusion", "MIS-TLIF", "spondylolisthesis", and "open transforaminal interbody fusion" as search subjects, Chinese and English databases were searched from the establishment of the database to March $15^{\text {th }}, 2021$ for RCTs on MI-TLIF in the treatment of spondylolisthesis databases included PubMed, Medline, Embase, Web of Sciences, Chinese Biolomedical Literature, Wanfang Chinese Biomedical Association Digital Journals, Wanfang Digital Journals FullText Database, and Weipu Chinese Sci-Tech Journals FullText Database.

The Boolean logic retrieval method was used to retrieve relevant references, and Rev Man 5.3 provided by the Cochrane system was used to evaluate the quality of the literature.

Some references were initially eliminated after reading the titles and abstracts, then a second screening was performed according to the inclusion criteria and exclusion criteria, and the reference was traced using a search engine. Finally, a third screening was conducted by reading the full text of the included literature.

\section{Inclusion and exclusion criteria}

References were selected as per the following inclusion criteria: (I) RCTs and retrospective case-control studies; (II) the subjects were spondylolisthesis patients for whom conservative treatment was ineffective for at least 5 weeks; (III) in the pathological control analysis, the index was reliable in the $95 \%$ confidence interval (CI); (IV) surgical methods were MI-TLIF and O-TLIF; (V) the study contained complete clinical basics data and observation indicators.

Exclusion criteria: (I) the patient had other systemic diseases; (II) the patient had received other types of surgery at the surgical site; (III) repeated published studies; (IV) related conference speeches, literature reviews, case study reports, lectures, and commentary literature; (V) studies with incomplete data.

Two senior experts performed the literature screening, and any inconsistency was resolved by discussion or inviting another expert to arbitrate.

\section{Observation indicators}

These were operation time, postoperative drainage volume, radiation exposure time, visual analogue scale (VAS) at the last follow-up, fusion rate at the last follow-up, and incidence of complications. 
Table 1 Newcastle-Ottawa Scale (NOS)

\begin{tabular}{|c|c|}
\hline Items & Evaluation results \\
\hline \multirow{2}{*}{$\begin{array}{l}\text { Is the case } \\
\text { diagnosed } \\
\text { appropriately? }\end{array}$} & Yes, the patient is diagnosed based on medical records or the doctor's own records, but there are no original records \\
\hline & No description \\
\hline \multirow[t]{2}{*}{$\begin{array}{l}\text { Case } \\
\text { representation }\end{array}$} & $\begin{array}{l}\text { Representative cases (cases with the target disease within a specified time; all cases in a specific hospital or clinic; a } \\
\text { random sample obtained from these cases) }\end{array}$ \\
\hline & There is potential selection bias or no description \\
\hline \multirow[t]{3}{*}{ Control selection } & Community control \\
\hline & Hospital control \\
\hline & No description \\
\hline
\end{tabular}

\section{Data extraction}

Two experts used unified Microsoft Excel to independently collate data and any inconsistency was resolved by discussion or inviting another expert to arbitrate. The following data were collated: (I) research title, research time, research type, follow-up time, and number of cases lost to follow-up; (II) first author's name, publication year, and publication name; (III) general information of the research object, including region, sample size, gender ratio, and age distribution; (IV) observation indicators, including operation time, postoperative drainage volume, and radiation exposure time.

\section{Risk of bias and quality assessment}

Two researchers conducted a risk assessment of bias simultaneously. Any inconsistencies were resolved via discussion or arbitration by a third expert. In this study, the Cochrane Collaboration for "bias risk assessment" was used for RCTs. The assessments of "low risk bias", "unclear", and "high risk bias" were made according to the four aspects of random method, blind method, allocation concealment, and the number of cases lost to follow up.

The Newcastle-Ottawa Scale (NOS) was used to evaluate the quality of the literature according to four aspects, namely, patient selection, comparability of the study, exposure assessment, and outcome. Each item accounted for 1 point, and the total score ranged from $0-9$ points. A score of 6-9 points was considered A-level research (low risk), 3-5 points were considered B-level research (unclear risk), and 0-2 was considered C-level research (high risk) (Table 1).

\section{Statistics}

Stata SE12.0 (College Station, USA) was used for statistical analysis and Rev Man 5.3 was used to assess the risk bias of the included references. Each effect was represented by a $95 \%$ CI. When $\mathrm{P}>0.01$ and $\mathrm{I}^{2}<50 \%$, the fixed effects model (FEM) was used for meta-analysis, and when $\mathrm{P}<0.01$ and $\mathrm{I}^{2}>50 \%$, the random effects model (REM) was used.

\section{Results}

\section{Basic information of the included references}

In total, 126 articles were obtained from the database and 139 articles were obtained from the register in this paper. By reading the abstract and title of the articles, 29 articles were repeatedly published, 43 articles were unqualified, and 32 articles were for other reasons, leaving 161 articles. After reading the full text, 63 articles with repeated subjects were eliminated, leaving 98 articles. 51 review reports were excluded, leaving 47 . The 35 articles that could not be extracted were excluded, and 12 articles were finally included in the study. Figure 1 shows the quality evaluation results of the NOS. It was noted that, 7 references $(58.33 \%)$ scored 6-9, 3 (25\%) scored 3-5, and 2 (16.67\%) scored 0-2 (Figure 2).

A total of 842 cases were involved in the 12 references. 


\section{Identification of studies via databases and registers}

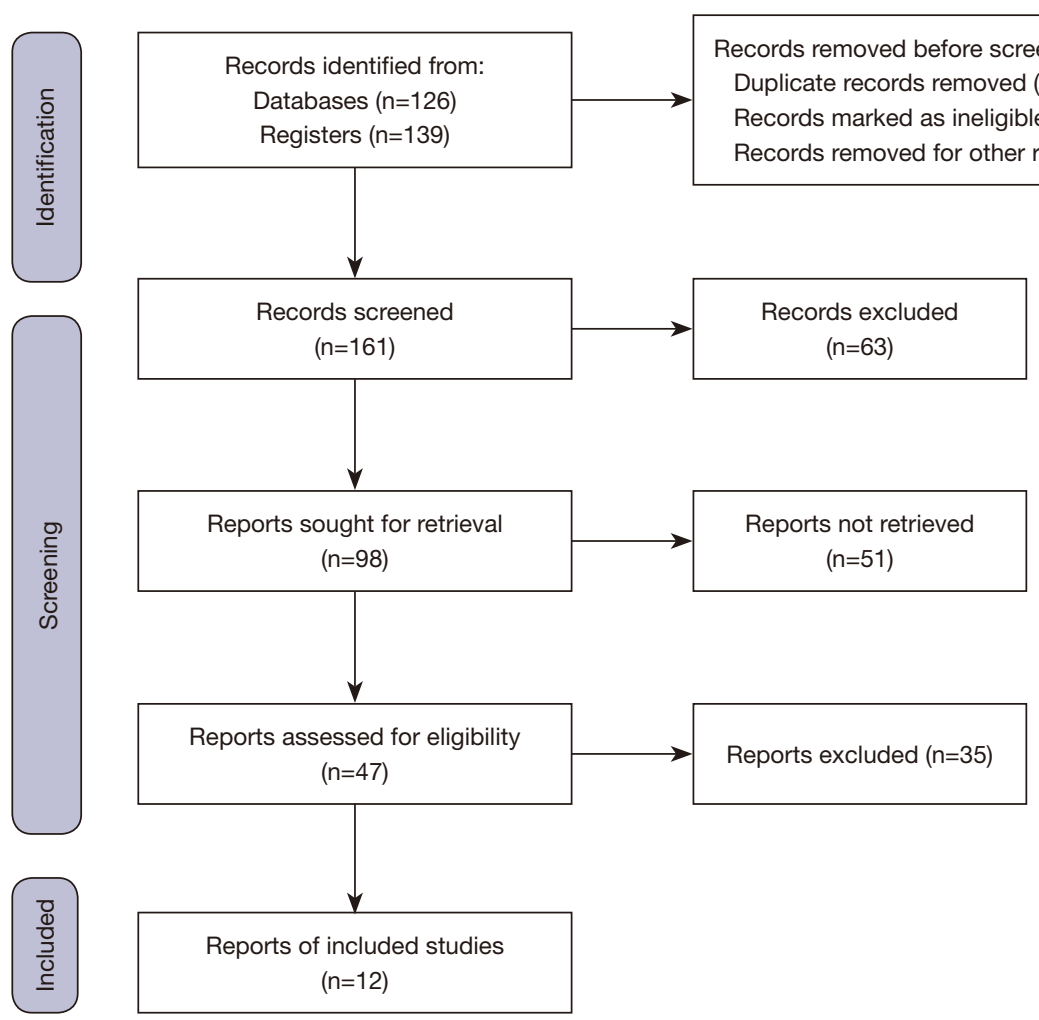

Figure 1 Flow chart showing the retrieval process.

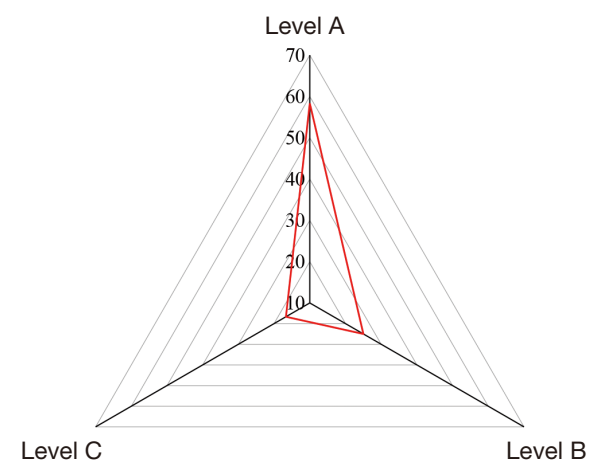

Figure 2 NOS results. NOS, Newcastle-Ottawa Scale.

All were small-sample studies, with the sample size ranging from 49 to 102 cases, and all research subjects were over 20 years old. All 12 references described the sample size, gender ratio, age, operation time, postoperative drainage volume, radiation exposure time, Oswestry disability index (ODI) score, VAS at the last follow-up, fusion rate at the last follow-up, and incidence of complications. Table 2 shows the basic characteristics of the included literature.

\section{Risk bias assessment}

Figures 3,4 show the multiple risk bias results of the included references assessed by Rev Man 5.3. Of the 12 RCTs in this study, four (9-12) described the correct random allocation method, accounting for $33.33 \%$, and two $(13,14)$ described the concealment allocation in detail, accounting for $16.67 \%$. Of the 12 RCTs, only one (15) of 12 used the blind method, accounting for $8.33 \%$.

\section{Operation time}

Figure 5 is a forest plot of the operation time. Of the 12 RCTs, eight described the mean difference (MD) and standard deviation (SD) of the operation time in detail, and the heterogeneity results showed that $\mathrm{I}^{2}=0 \%<50 \%$, 
Table 2 Basic characteristics of included literature

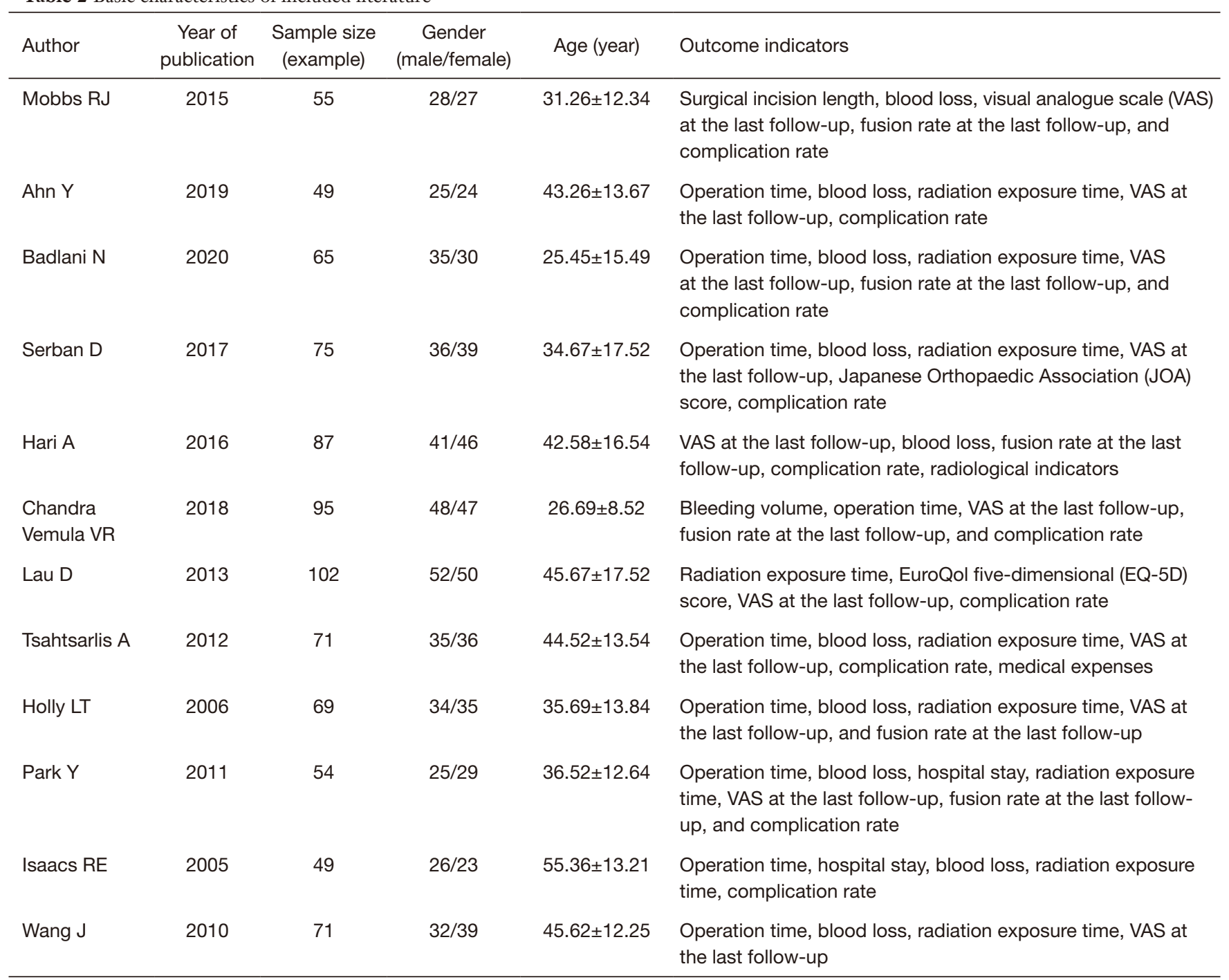

$\mathrm{P}=0.70$, indicating that there was no heterogeneity. REM was then used for analysis, which showed there was no significant difference in the operation time between MITLIF and O-TLIF for spondylolisthesis [MD $=-19.99$, 95\% CI: $(-23.95,-16.04), \mathrm{P}<0.00001]$, indicating there was no significant difference in the operation time for the two methods.

\section{Intraoperative blood loss}

Figure 6 shows the meta-analysis results of intraoperative blood loss. Of the 12 RCTs, 11 described the MD and SD of intraoperative blood loss in detail, while heterogeneity results showed that $\mathrm{I}^{2}=93 \%>50 \%, \mathrm{P}<0.00001$, indicating obvious heterogeneity. RCT was then used for analysis and showed the difference in intraoperative blood loss between MI-TLIF and O-TLIF was statistically significant $[\mathrm{MD}=-349.35,95 \%$ CI: $(-410.66,-288.03), \mathrm{P}<0.00001]$. Compared with O-TLIF, MI-TLIF could reduce intraoperative blood loss.

\section{Intraoperative radiation exposure time}

Figure 7 shows the meta-analysis results of intraoperative radiation exposure time, and of the 12 RCTs, nine described the MD and SD in detail. The heterogeneity results showed that $\mathrm{I}^{2}=94 \%>50 \%, \mathrm{P}<0.00001$, indicating obvious heterogeneity. REM was then used and showed 


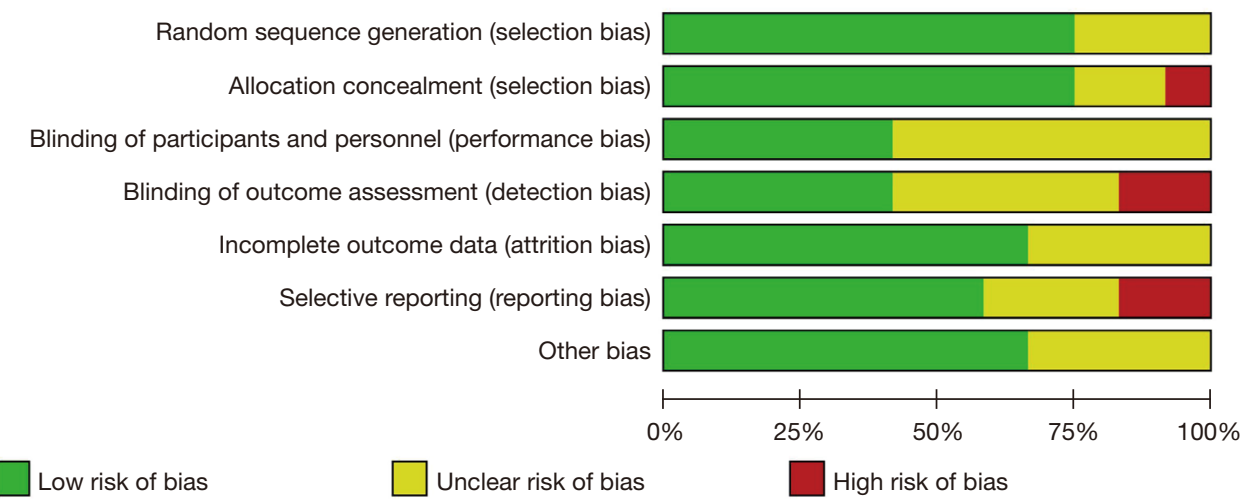

Figure 3 Risk bias assessment.

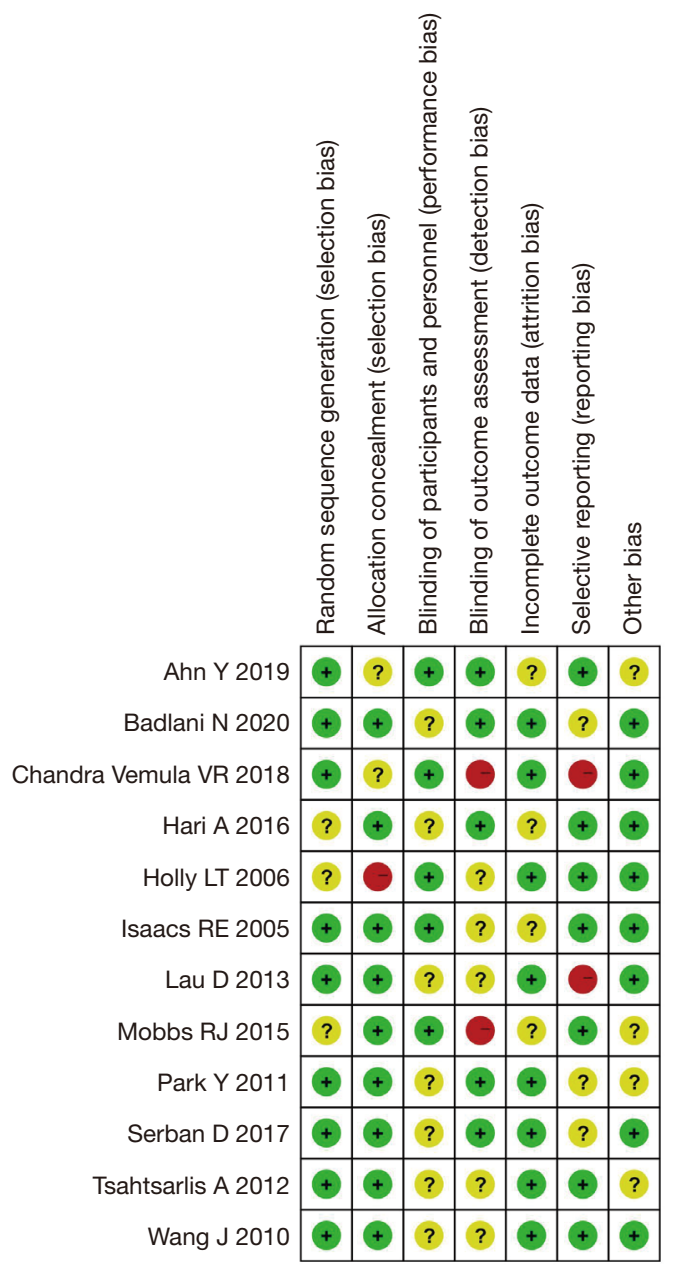

Figure 4 Multiple risk bias assessment. + = low risk bias; - = high risk bias; ? = unclear. the difference in intraoperative radiation exposure time between MI-TLIF and O-TLIF was statistically significant [MD $=35.72,95 \%$ CI: $(28.11,43.33), \mathrm{P}<0.00001]$, with MITLIF's radiation exposure time significantly reduced in comparison to OTLIF.

\section{Fusion rate at the last follow-up}

Figure 8 shows the meta-analysis results of the fusion rate at the last follow-up, and of the 12 RCTs, six described the mean and SD in detail. The heterogeneity results showed that $\mathrm{I}^{2}=0 \%<50 \%, \mathrm{P}=0.99$, indicating there was no heterogeneity. FEM was then used for analysis and showed the fusion rate of MI-TLIF and O-TLIF was not statistically significant [odds ratio $(\mathrm{OR})=0.80,95 \% \mathrm{CI}$ : $(0.31,2.06), \mathrm{P}=0.64]$, indicating the two treatment methods had similar fusion rates.

\section{VAS score}

Figures 9,10 show the meta-analysis results of VAS scores, and of the 12 RCTs, seven described the MD and SD of pain improvement in detail. The heterogeneity results of the VAS score showed that $\mathrm{I}^{2}=96 \%>50 \%, \mathrm{P}<0.00001$, indicating obvious heterogeneity. REM was then used for analysis, and showed a statistically significant difference in VAS scores before and after O-TLIF at the last follow-up $[\mathrm{MD}=6.04,95 \% \mathrm{CI}:(4.94,7.14), \mathrm{P}<0.00001]$, and in VAS scores before and after MI-TLIF at the last follow-up [MD $=5.72,95 \%$ CI: $(4.83,6.62), \mathrm{P}<0.00001]$. 


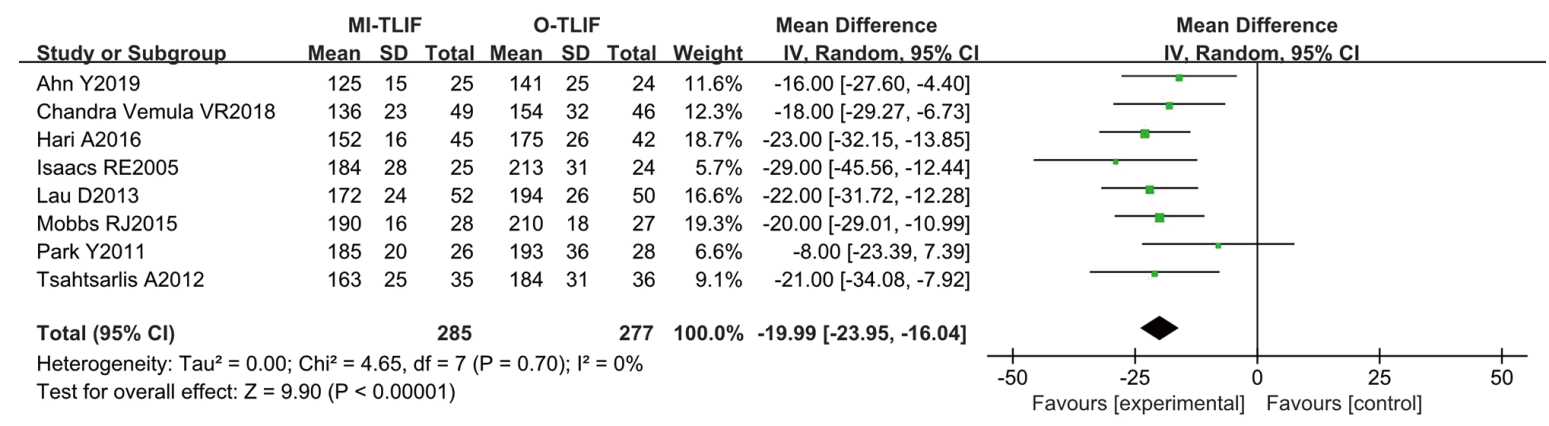

Figure 5 Forest plot of operation time. MI-TLIF, minimally invasive transforaminal interbody fusion; O-TLIF, open transforaminal interbody fusion; SD, standard deviation; CI, confidence interval.

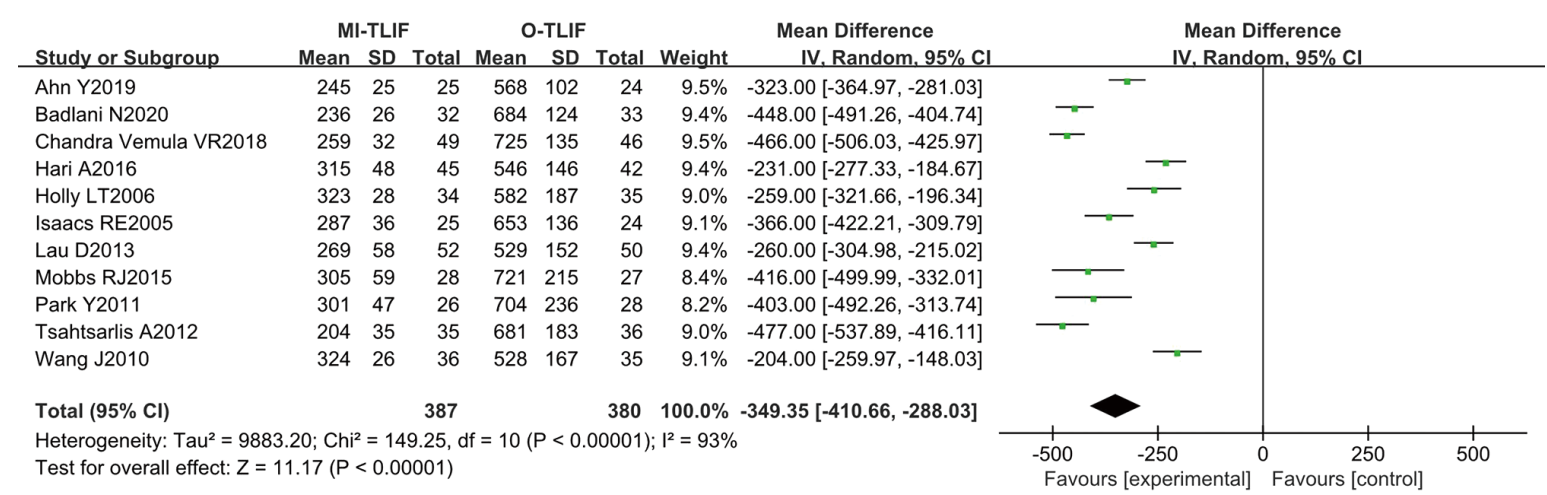

Figure 6 Meta-analysis results of intraoperative blood loss. MI-TLIF, minimally invasive transforaminal interbody fusion; O-TLIF, open transforaminal interbody fusion; SD, standard deviation; CI, confidence interval.

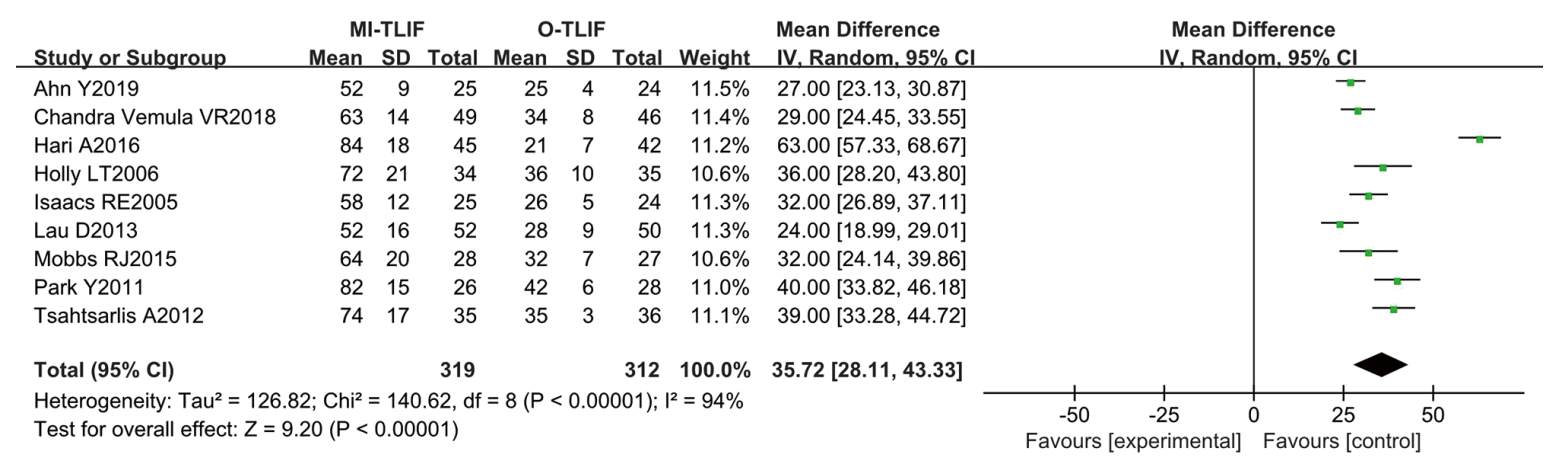

Figure 7 Meta-analysis results of intraoperative radiation exposure time. MI-TLIF, minimally invasive transforaminal interbody fusion; O-TLIF, open transforaminal interbody fusion; SD, standard deviation; CI, confidence interval.

\section{Complication rate}

Figure 11 shows the meta-analysis results of the incidence of complications, and of the 12 RCTs, 10 described the MD and $\mathrm{SD}$ of the complication rate in detail. The heterogeneity results showed that $\mathrm{I}^{2}=0 \%<50 \%, \mathrm{P}=0.58$, indicating no heterogeneity. FEM was then used for analysis, and showed the complication rate difference between MI-TLIF and O-TLIF treatment was statistically significant $[\mathrm{OR}=0.48$, 95\% CI: (0.30, 0.76), P=0.002] Compared to O-TLIF, MI- 


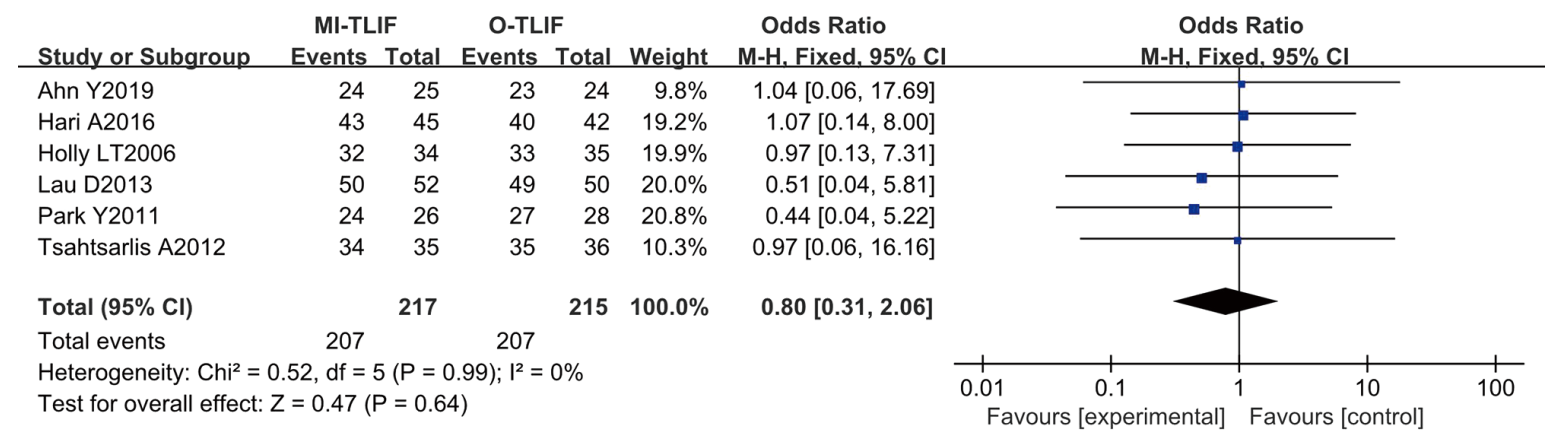

Figure 8 Meta-analysis results of the fusion rate at the last follow-up. MI-TLIF, minimally invasive transforaminal interbody fusion; O-TLIF, open transforaminal interbody fusion; CI, confidence interval.

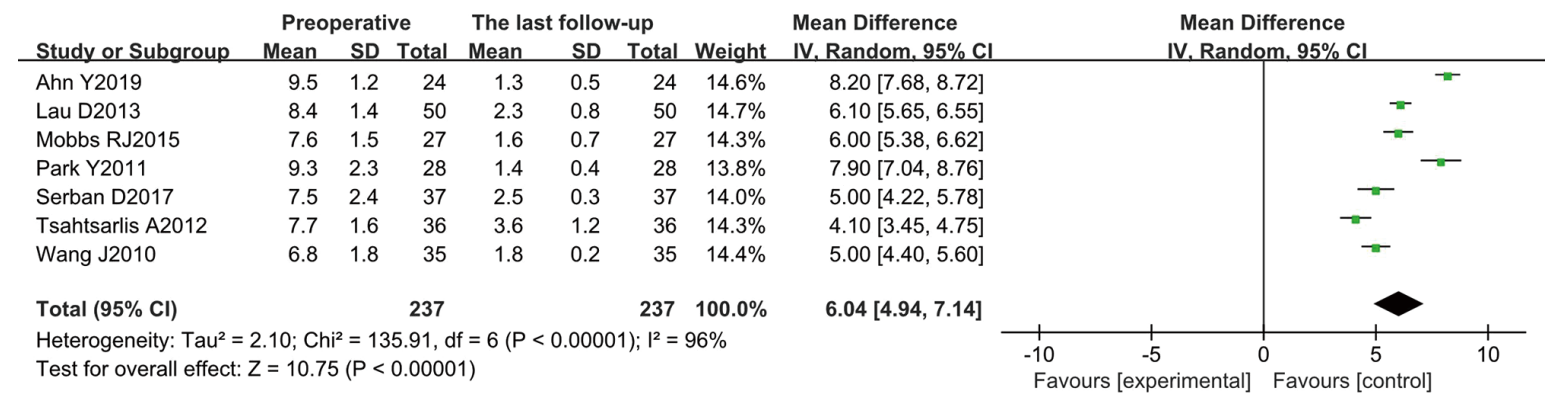

Figure 9 Meta-analysis results of VAS scores before and after O-TLIF at the last follow-up. VAS, visual analogue scale; O-TLIF, open transforaminal interbody fusion; SD, standard deviation; CI, confidence interval.

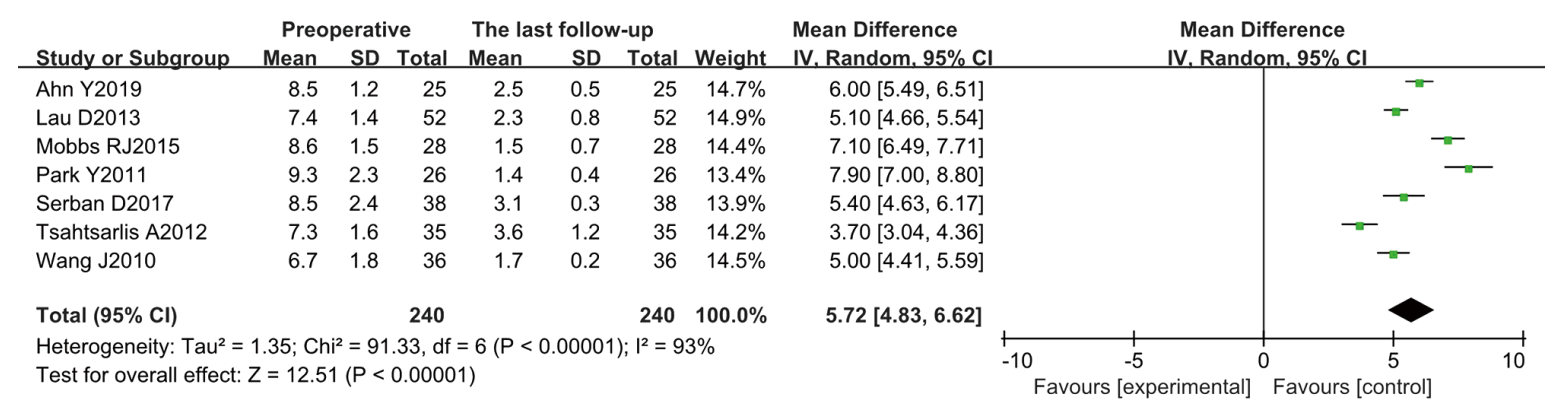

Figure 10 Meta-analysis results of visual pain scores before and after MI-TLIF at the last follow-up. MI-TLIF, minimally invasive transforaminal interbody fusion; SD, standard deviation; CI, confidence interval.

TLIF could reduce the incidence of complications.

\section{Publication of bias}

Figure 12 is a funnel chart of the publication bias of the included literature. This shows the circles in some studies were basically symmetrical along the center line, there was no bias in publications, and the conclusions obtained were credible.

\section{Discussion}

Spondylolisthesis is a common spinal disease, and foraminal interbody fusion is a standard treatment 


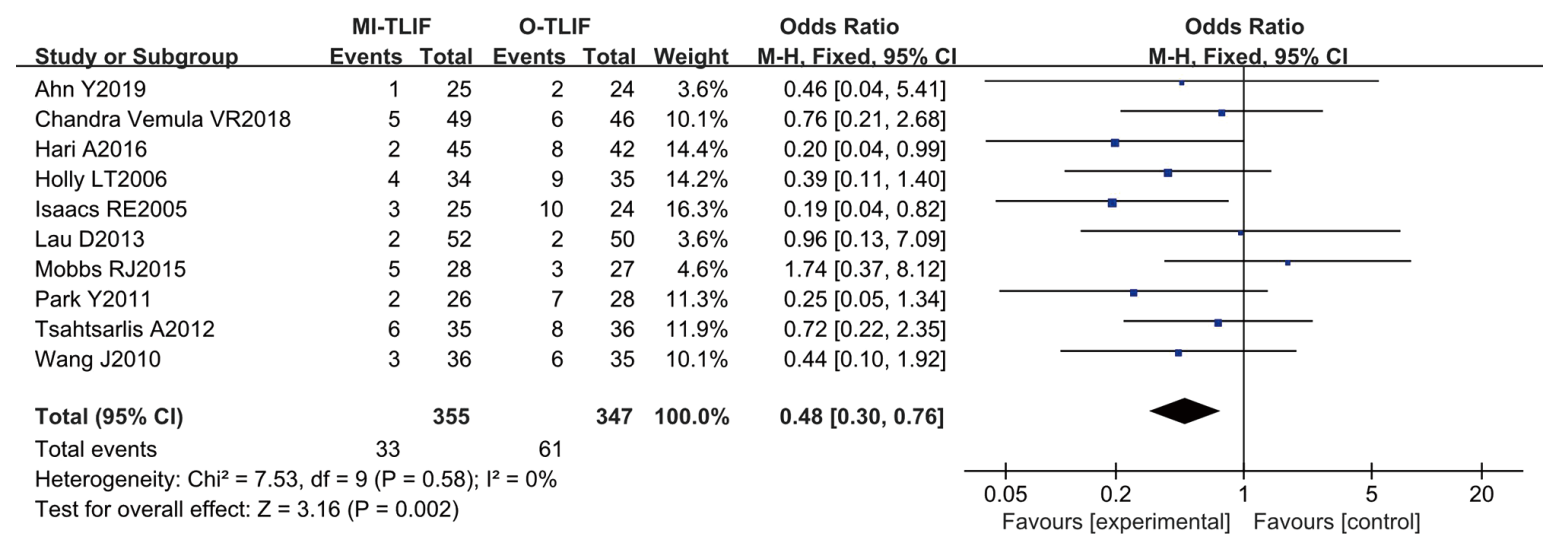

Figure 11 Meta-analysis results of complication rate. MI-TLIF, minimally invasive transforaminal interbody fusion; O-TLIF, open transforaminal interbody fusion; CI, confidence interval.

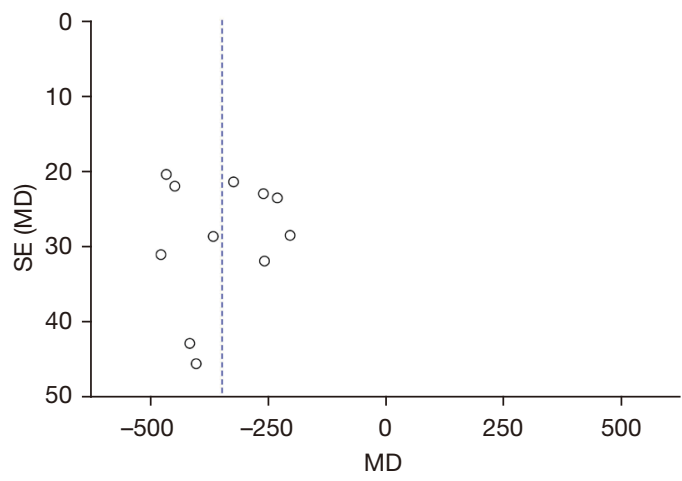

Figure 12 Funnel chart of the publication bias of the included literature. This indicated the included literature was reliable, and risk bias was not a key factor affecting the conclusion. SE, standard error; $\mathrm{MD}$, mean difference.

method (16). Clinically, TLIF has been widely used for the treatment of degenerative diseases of the lumbar spine, and compared with posterior interbody fusion, can reduce the risk of nerve injury and maintain the stability of the posterior column (17). MI-TLIF is used to treat degenerative spondylolisthesis and spondylolisthesis. For spondylolisthesis, the bone in the spondylolisthesis is damaged, and postoperative treatment such as reduction, bone grafting, fusion, and repair is needed, resulting in more blood loss and longer operation time in spondylolisthesis than in degenerative spondylolisthesis. However, traditional intervertebral transforaminal fusion may lead to multifidus muscle dissection and muscle nerve atrophy, while MI-TLIF results in less damage to the paraspinal muscle, reduces avascular necrosis and aseptic inflammation, and maintains the stability of the spine. MITLIF is a new interbody fusion technology developed in the past decade. Compared with traditional open surgery, MITLIF has the advantage of avoiding a wide range of muscle and soft tissue dissection or cutting, significantly reducing intraoperative bleeding, postoperative pain, and hospital stay, and reducing nerve root stimulation and postoperative scar formation. Because of adequate bone contact surface and abundant blood supply, intervertebral bone grafting can achieve satisfactory fusion rates.

A minimally invasive approach reduces time in the operating room, minimizes blood loss and tissue damage, and helps minimize pain during healing. It also speeds recovery time and reduces the chance of complications (18). While there was no significant difference in operation time between MI-TLIF and O-TLIF for spondylolisthesis $[\mathrm{MD}=-19.99,95 \% \mathrm{CI}:(-23.95,-16.04), \mathrm{P}<0.00001]$, there was in intraoperative blood loss $[\mathrm{MD}=-349.35,95 \% \mathrm{CI}$ : (-410.66, -288.03), $\mathrm{P}<0.00001]$. This suggests the operation time of the two surgical treatments was not significantly different, but MI-TLIF could significantly reduce the amount of intraoperative blood loss, which was consistent with the results of Kirnaz et al. [2020] (19). This may be associated with the small surgical incisions used in MITLIF and low tissue damage.

There was a statistically significant difference in the VAS before and after O-TLIF [MD $=6.04,95 \%$ CI: $(4.94,7.14)$, $\mathrm{P}<0.00001]$ and after MI-TLIF $[\mathrm{MD}=5.72,95 \% \mathrm{CI}$ : $(4.83$, 6.62), $\mathrm{P}<0.00001]$ at the last follow-up. This showed that both treatment methods could significantly improve the 
pain of patients. The complication rate difference between MI-TLIF and O-TLIF was also statistically significant [OR $=0.48,95 \% \mathrm{CI}:(0.30,0.76), \mathrm{P}=0.002]$, and compared with O-TLIF, MI-TLIF could reduce the incidence of complications. The occurrence of complications may be related to factors such as the technological difficulties of minimally invasive surgery and the long learning curve $(20,21)$.

A meta-analysis was conducted on MI-TLIF in the treatment of spondylolisthesis, and a funnel chart was drawn. This showed there was no bias in publication, and the conclusions obtained were credible, and risk bias was not the main factor affecting the conclusion.

\section{Conclusions}

In this meta-analysis, the compound logic search method was used to retrieve 12 RCTs on MI-TLIF in the treatment of spondylolisthesis. It was confirmed that MI-TLIF can significantly reduce intraoperative blood loss, mitigate patient pain, and reduce the incidence of complications without increasing the operation time. A limitation of this study is that the number of studies on MI-TLIF was limited, which may reduce the power of the study. Multi-center and large-scale clinical data are needed to further study the effectiveness of MI-TLIF. In conclusion, this study confirmed MI-TLI provides a safe and effective surgical method for the treatment of spondylolisthesis.

\section{Acknowledgments}

Funding: None.

\section{Footnote}

Reporting Checklist: The authors have completed the PRISMA reporting checklist. Available at https://dx.doi. org/10.21037/apm-21-2137

Conflicts of Interest: All authors have completed the ICMJE uniform disclosure form (available at https://dx.doi. org/10.21037/apm-21-2137). The authors have no conflicts of interest to declare.

Ethical Statement: The authors are accountable for all aspects of the work in ensuring that questions related to the accuracy or integrity of any part of the work are appropriately investigated and resolved.

Open Access Statement: This is an Open Access article distributed in accordance with the Creative Commons Attribution-NonCommercial-NoDerivs 4.0 International License (CC BY-NC-ND 4.0), which permits the noncommercial replication and distribution of the article with the strict proviso that no changes or edits are made and the original work is properly cited (including links to both the formal publication through the relevant DOI and the license). See: https://creativecommons.org/licenses/by-nc-nd/4.0/.

\section{References}

1. Mobbs RJ, Phan K, Malham G, et al. Lumbar interbody fusion: techniques, indications and comparison of interbody fusion options including PLIF, TLIF, MI-TLIF, OLIF/ATP, LLIF and ALIF. J Spine Surg 2015;1:2-18.

2. Kolcun JP, Brusko GD, Wang MY. Endoscopic transforaminal lumbar interbody fusion without general anesthesia: technical innovations and outcomes. Ann Transl Med 2019;7:S167.

3. Badlani N, Yu E, Kreitz T, et al. Minimally Invasive Transforaminal Lumbar Interbody Fusion (TLIF). Clin Spine Surg 2020;33:62-4.

4. Foley KT, Lefkowitz MA. Advances in minimally invasive spine surgery. Clin Neurosurg 2002;49:499-517.

5. Serban D, Calina N, Tender G. Standard versus minimally invasive transforaminal lumbar interbody fusion: a prospective randomized study. Biomed Res Int 2017;2017:7236970.

6. Hari A, Krishna M, Rajagandhi S, et al. Minimally invasive transforaminal lumbar interbody fusion-indications and clinical experience. Neurol India 2016;64:444-54.

7. Chandra Vemula VR, Prasad BC, Jagadeesh MA, et al. Minimally invasive transforaminal lumbar interbody fusion using bone cement-augmented pedicle screws for lumbar spondylolisthesis in patients with osteoporosis. Case series and review of literature. Neurol India 2018;66:118-25.

8. Lau D, Ziewacz J, Park P. Minimally invasive transforaminal lumbar interbody fusion for spondylolisthesis in patients with significant obesity. J Clin Neurosci 2013;20:80-3.

9. Tsahtsarlis A, Wood M. Minimally invasive transforaminal lumbar interbody fusion and spondylolisthesis. J Clin Neurosci 2012;19:858-61.

10. Holly LT, Schwender JD, Rouben DP, et al. Minimally invasive transforaminal lumbar interbody fusion: 
indications, technique, and complications. Neurosurg Focus 2006;20:E6.

11. Park Y, Ha JW, Lee YT, et al. Surgical outcomes of minimally invasive transforaminal lumbar interbody fusion for the treatment of spondylolisthesis and degenerative segmental instability. Asian Spine J 2011;5:228-36.

12. Isaacs RE, Podichetty VK, Santiago P, et al. Minimally invasive microendoscopy-assisted transforaminal lumbar interbody fusion with instrumentation. J Neurosurg Spine 2005;3:98-105.

13. Wang J, Zhou Y, Zhang ZF, et al. Comparison of onelevel minimally invasive and open transforaminal lumbar interbody fusion in degenerative and isthmic spondylolisthesis grades 1 and 2. Eur Spine J 2010;19:1780-4.

14. Khalifeh JM, Dibble CF, Stecher P, et al. Transfacet minimally invasive transforaminal lumbar interbody fusion with an expandable interbody device-part I: 2-dimensional operative video and technical report. Oper Neurosurg (Hagerstown) 2020;19:E473-9.

15. Massel DH, Mayo BC, Long WW, et al. Minimally invasive transforaminal lumbar interbody fusion: comparison of grade I versus grade II isthmic spondylolisthesis. Int J Spine Surg 2020;14:108-14.

16. Wipplinger C, Melcher C, Hernandez RN, et al. "One and a half" minimally invasive transforaminal lumbar interbody fusion: single level transforaminal lumbar interbody fusion with adjacent segment unilateral laminotomy for bilateral decompression for spondylolisthesis with bisegmental

Cite this article as: Chen Z, Wu W, Xiong H, Li G, Zhang W, Gao Y, Wang M. Systematic review and meta-analysis of the therapeutic effects of minimally invasive transforaminal interbody fusion on spondylolisthesis. Ann Palliat Med 2021;10(9):9848-9858. doi: 10.21037/apm-21-2137 stenosis. J Spine Surg 2018;4:780-6.

17. Chan AK, Bisson EF, Bydon M, et al. A comparison of minimally invasive transforaminal lumbar interbody fusion and decompression alone for degenerative lumbar spondylolisthesis. Neurosurg Focus 2019;46:E13.

18. Khalifeh JM, Dibble CF, Stecher P, et al. Transfacet minimally invasive transforaminal lumbar interbody fusion with an expandable interbody device-part II: consecutive case series. Oper Neurosurg (Hagerstown) 2020;19:518-29.

19. Kirnaz S, Navarro-Ramirez R, Wipplinger C, et al. Minimally invasive transforaminal lumbar interbody fusion using 3-dimensional total navigation: 2-dimensional operative video. Oper Neurosurg (Hagerstown) 2020;18:E9-10.

20. Qin R, Liu B, Zhou P, et al. Minimally invasive versus traditional open transforaminal lumbar interbody fusion for the treatment of single-level spondylolisthesis grades 1 and 2: a systematic review and meta-analysis. World Neurosurg 2019;122:180-9.

21. Goh GS, Tay YWA, Liow MHL, et al. Elderly patients undergoing minimally invasive transforaminal lumbar interbody fusion may have similar clinical outcomes, perioperative complications, and fusion rates as their younger counterparts. Clin Orthop Relat Res 2020;478:822-32.

(English Language Editor: B. Draper) 